\title{
Nonautonomous difference equations: Open problems and conjectures
}

\author{
Saber N. Elaydi \\ Department of Mathematics, Trinity University, \\ San Antonio, Texas 78212
}

\section{Introduction}

Autonomous difference equations of the form $x_{n+1}=f\left(x_{n}\right)$ may model populations of species with nonoverlaping generations such as fish, orchard pests, etc. The drawback of such models is that they do not account for environmental fluctuations or seasonal changes. Hence we are led to nonautonomous difference equations of the form $x_{n+1}=f_{n}\left(x_{n}\right), n \in \mathbb{Z}^{+}$. Our main focus in this note will be on periodic difference equations in which the sequence $f_{n}$ is periodic. Most of the open problems and conjectures in this part are motivated by recent work by Elaydi and Sacker [3], Elaydi and Yakubu [4] [5], and Elaydi [2]. The second part of the paper discusses the connection between a nonautonomous difference equation and its limiting equation. We present here several conjectures and open problems pertaining to the properties of omega limit sets (see Kempf [7]) and the question of lifting properties from the limiting equation to the original equation. For the convenience of the reader we introduce in Section 4 some rudiments of the theory of skew-product dynamical systems [8].

\section{Periodic difference equations}

Consider the nonautonomous difference equation

$$
x_{n+1}=f\left(n, x_{n}\right)
$$


which may be written in the form

$$
x_{n+1}=f_{n}\left(x_{n}\right), \quad n \in \mathbb{Z}^{+}
$$

where $\mathbb{Z}^{+}$is the set of nonnegative integers and $f_{n}: X \rightarrow X$ is a continuous function on a metric space $X$. If $f_{n+p}=f_{n}$ for all $n \in \mathbb{Z}^{+}$, then Eq. (2.1) is said to be periodic of period $p$ or $p$-periodic. On the other hand, if $f_{n}=f$ for all $n \in \mathbb{Z}^{+}$, then Eq.(2.1) reduces to the autonomous difference equation

$$
x_{n+1}=f\left(x_{n}\right)
$$

The "semi flow" of equation (2.2) is defined by the double sequence

$$
\Phi\left(n, n_{0}\right)=f_{n+n_{0}-1} \circ f_{n+n_{0}-2} \circ \cdots \circ f_{n_{0}}, \quad \Phi\left(n_{0}, n_{0}\right)=i d
$$

If $n_{0}=0$, then we write $\Phi(n, 0)=\Phi_{n}$.

The orbit of $x_{0}$ is given by

$$
\mathcal{O}\left(x_{0}\right)=\left\{\Phi_{n}\left(x_{0}\right): n \in \mathbb{Z}^{+}\right\} .
$$

Elaydi and Yakubu [4] showed that a globally asymptotically stable periodic cycle of Eq.(2.3) must be a fixed point if the space $X$ is connected. A natural question is whether or not this theorem extends to nonautonomous difference equations. A definitive answer was recently provided by Elaydi and Sacker [3].

Theorem 2.1. [3] Suppose that $f_{n+p}=f_{n}$ for all $n \in \mathbb{Z}^{+}$, for some $p \in \mathbb{Z}^{+}$. Then a globally asymptotically stable periodic cycle of Eq.(2.1) must be of period $p$ or a divisor of $p$.

Theorem 2.2. [3] Given any two positive integers $r$ and $k$ such that $r$ is a divisor of $k$. Then there exists a nonautonomous periodic difference equation with minimum period $k$ and which has a globally asymptotically stable periodic orbit with minimal period $r$

The following example illustrates the above theorems. 
Example 2.3. [3] Consider the equation

$$
\begin{aligned}
x_{n+1} & =f_{n}\left(x_{n}\right) \\
\text { with } f_{0}(x) & =1+\alpha x \\
f_{1}(x) & =-\alpha+\alpha x \\
f_{2}(x) & =1+\beta x \\
f_{3}(x) & =-\beta+\beta x \\
f_{n} & =f \bmod 4
\end{aligned}
$$

The 2-cycle $\{0,1\}$ is globally asymptotically stable, while the difference equation is 4-periodic.

A periodic solution of (2.1) may have a periodic solution whose period is unrelated to $p$ as demonstrated by the following example.

Example 2.4. Consider the system of difference equations.

$$
\begin{aligned}
& x_{n+1}=x_{n}-y_{n}+\left(x_{n}^{2}+y_{n}^{2}-1\right) \cos \frac{2 \pi}{3} n \\
& y_{n+1}=x_{n}+y_{n}+\left(x_{n}^{2}+y_{n}^{2}-1\right) \sin \frac{2 \pi}{3} n
\end{aligned}
$$

This is a periodic system with period $p=3$. However, the solution $x_{n}=$ $\cos \frac{n \pi}{4}, y_{n}=\sin \frac{n \pi}{4}$ is of period 8. Notice that, by virtue of Theorem 2.1 this 8-periodic orbit cannot be globally asymptotically stable.

Such periodic orbits will not be considered and we will be interested in periodic orbits of period $p$ or in "subharmonics" whose minimal period is a mltiple of $p$ or in "subharmonics" whose period is a divisor of $p$.

Lemma 2.5. A solution of $\varphi(n)=\Phi_{n}\left(x_{0}\right)$ is p-periodic if and only if

$$
\Phi_{p}\left(x_{0}\right)=x_{0} .
$$

Proof. Clearly if $\varphi(n)$ is $p$-periodic, then $\Phi_{p}\left(x_{0}\right)=x_{0}$. Conversely, define $\psi(n)=\varphi(n+p)$.

Then

$$
\begin{aligned}
\psi(n+1) & =\varphi(n+1+p) \\
& =f_{n+p}(\varphi(n+p)) \\
& =f_{n}(\psi(n))
\end{aligned}
$$


Consequently, $\psi(n)$ is a solution of equation Eq.(2.1). Moreover, $\psi(0)=$ $\varphi(p)=\varphi(0)$ which implies by uniqueness of solutions that $\psi(n)=\varphi(n)$. Hence $\varphi(n+p)=\varphi(n)$ for all $n \in \mathbb{Z}^{+}$.

We now turn our attention to scalar periodic difference equations.

Theorem 2.6. Eq. (2.1) has a p-periodic solution if and only if the operator $\Phi_{p}: X \rightarrow X$ has a fixed point $x_{o}$. If $x_{0}$ is a fixed point of $\Phi_{p}$, then $\varphi\left(n, x_{0}\right)$ is a periodic solution of Eq.(2.1).

Theorem 2.7. Suppose that Eq.(2.1) has a bounded solution. Then it has a p-periodic solution.

Proof. Let $x_{n}=\Phi_{n}\left(x_{0}\right)$ be a bounded solution of Eq. (2.1). Consider the point $x_{p}=\Phi_{p}\left(x_{0}\right)$. Then either $x_{p}>x_{0}$ or $x_{p}<x_{0}$. For if $x_{p}=x_{0}$ we then would have a $p$-periodic solution. Assume that $x_{p}>x_{0}$. If $x_{2 p}=\Phi_{p}\left(x_{p}\right)<x_{p}$, then Eq. (2.1) has a $p$-periodic solution. So assume that the sequence $\Phi_{n p}\left(x_{0}\right)$ is monotonically increasing. And since it is bounded it converges to a point $\bar{z}$ which is clearly a $p$-periodic solution of Eq.(2.1).

Remark 2.8. The main difficulty in corollary 2.7 is that it does not distinguish non-constant periodic solutions from equilibria (fixed points). One exception is the periodically forced autonomous systems of the form

$$
x_{n+1}=f\left(x_{n}\right)+b(n)
$$

where $b(n+p)=b(n)$ for all $n \in \mathbb{Z}^{+}$. If $b$ is nonconstant, then equation (2.5) has no equilibrium points.

Consider the logistic map

$$
x_{n+1}=\mu x_{n}\left(1-x_{n}\right)
$$

It is known that the positive fixed point of Eq.(2.6) $x^{*}=\frac{\mu-1}{\mu}$ is globally asymptotically stable if $1<\mu \leq 3$.

Suppose now we replace $\mu$ with a periodic sequence $\mu_{n}$ with period $p$, i.e.,

$$
\mu_{n+p}=\mu_{n} \text { for all } n \in \mathbb{Z}^{+} .
$$

We than have

$$
x_{n+1}=\mu_{n} x_{n}\left(1-x_{n}\right)
$$


Open Problem 2.9. Find conditions on $m u_{n}$ under which Eq.(2.7) has a globally asymptotically stable p-cycle

The Ricker's map given by $R(x)=x e^{\beta-x}$ has a globally asymptotically stable fixed $\beta$ if $0<\beta \leq 2$. The corresponding p-periodic difference equation is given by

$$
x_{n+1}=x_{n} e^{\beta_{n}-x_{n}}
$$

Open Problem 2.10. Find conditions under which Eq.(2.8) has a globally asymptotically p-cycle.

A more general open problem concerns itself with difference equations with a parameter such as

$$
x_{n+1}=f\left(x_{n}, \alpha\right)
$$

Open Problem 2.11. Suppose that Eq. (2.9) has a globally asymptotically stable fixed point in a positive cone in $\mathbb{R}^{m}$ for $c<\alpha<d$. Find conditions under which the equation

$$
x_{n+1}=f\left(x_{n}, \alpha_{n}\right)
$$

where $\alpha_{n+p}=\alpha_{n}$. has a globally asymptotically stable $r$-cycle where $r$ is a divisor of $p$.

\section{Extension of Sharkovsky's Theorem to pe- riodic difference equations}

Consider the following ordering of the natural numbers:

$$
\begin{array}{cc}
3 \triangleright 5 \triangleright 7 \triangleright \ldots & 2 \times 3 \triangleright 2 \times 5 \triangleright 2 \times 7 \triangleright \ldots \\
\text { odd integers } & 2 \times \text { odd integers } \\
& \\
2^{2} \times 3 \triangleright 2^{2} \times 5 \triangleright 2^{2} \times 7 \triangleright \ldots & 2^{n} \times 3 \triangleright 2^{n} \times 5 \triangleright 2^{n} \times 7 \triangleright \ldots \\
2^{2} \times \text { odd integers } & 2^{n} \times \text { odd integers } \\
\ldots \ldots \ldots & \cdots \triangleright 2^{n} \triangleright \ldots \triangleright 2^{3} \triangleright 2^{2} \triangleright 2 \triangleright 1 \\
& \text { powers of } 2 .
\end{array}
$$


This exhausts all the natural numbers. This is called the Sharkovsky's ordering of the natural numbers. Sharkovsky's Theorem (f. Sharkovsky [9], Elaydi [1]) is

Theorem 3.1. Suppose $f: \mathbb{R} \rightarrow \mathbb{R}$ is continuous and $f$ has a periodic point of minimal period $k$. If $k \triangleright r$ in the Sharkovsky's ordering, then $f$ also has a periodic point of period $r$.

It is of great interest to extend this remarkable result to periodic difference equations.

Conjecture 3.2. Suppose that $f_{n+p}=f_{n}$ in Eq.(2.1) and $f_{n}: \mathbb{R} \rightarrow \mathbb{R}$ is continuous, $n \in \mathbb{Z}^{+}$. Suppose that Eq.(2.1) has a periodic point of minimal period $k$. Let $s=[p, k]$, be the least common divisor and let $\ell=s / k$. If $\ell \triangleright r$ in the Sharkovsky's ordering, then $f$ also has a periodic point of period $r$. In particular, if $p$ and $k$ are relatively prime, and $k$ rhdr, then $f$ has a point of period $r$.

A converse of Sharkovsky's theorem was proved in Elaydi [2]

Theorem 3.3. Given any two positive integers $r$ and $k$ such as $r \triangleright k$ in the Sharkovsky's order. Then there exists a continuous function $f: \mathbb{R} \rightarrow \mathbb{R}$ which has a point of period $k$ but no points of period $r$.

Open Problem 3.4. Extend the above theorem to periodic difference equations.

In the next section we introduce the notion of a skew-product dynamical system which is, in our view, the main tool in studying nonautonomous difference equations. Notice that, unlike autonomous difference equations, nonautonomous difference equations do not give rise to a dynamical system. Such defect is remedied by defining a dynamical system on an enlarged space. This is in essence the main idea behind the notion of skew-product dynamical systems. In [8], Sacker an Sell developed the theory of skew-product dynamical systems and applied it to the study of nonautonomous differential equations. In [3], Elaydi and Sacker is developing the theory of discrete skew-product semidynamical systems and applying it to the study of nonautonomous difference equations. We now introduce some rudiments of the theory of skew-product dynamical systems. 


\section{Skew-product dynamical system}

Definition 4.1. Let $X$ and $Y$ be two metric spaces. A dynamical system $\pi$ on a product space $X \times Y$ is said to be a skew-product dynamical system if

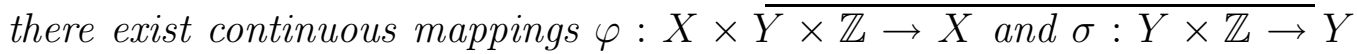
such that

$$
\pi(x, y, n)=(\varphi(x, y, n), \sigma(y, n)) .
$$

If $\mathbb{Z}$ is replaced by $\mathbb{Z}^{+}$, then $\pi$ is called a skew-product semi-dynamical system.

Now let us construct a skew-product semi-dynamical system for the periodic nonautonomous difference equation Eq.(2.1)

We let $Y=\left\{f_{n}: n \in \mathbb{Z}^{+}\right\}$. Now for $f_{i}$, we define $\sigma^{r}\left(f_{i}\right)=f_{i+r}$ as the shift map.

The map $\varphi$ is defined as

$$
\varphi\left(x_{0}, f_{i}, n\right)=\Phi(n, i) x_{0}
$$

Hence

$$
\pi\left(\left(x_{0}, f_{i}\right), n\right)=\left(\Phi(n, i) x_{0}, f_{i+n}\right)
$$

For the general construction of skew-product dynmaical systems we refer the reader to Elaydi and Sacker[3]. If $p$ is the projection map, then the following diagram commutes.

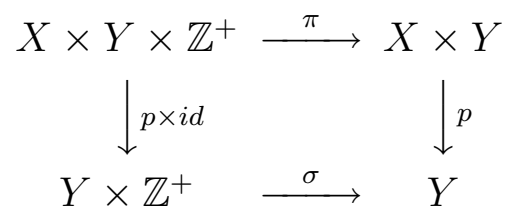

Hence $\pi$ is "fiber preserving": For every $(y, n) \in Y \times \mathbb{Z}^{+}$, the fiber over $(y, n)$ is defined to be $\left(p^{-1} \times i d\right)(y, n)$ and fiber preserving means that $\pi\left(\left(p^{-1} \times\right.\right.$ $i d)(y, n)) \subset p^{-1}(\sigma(y, n))$.

Open Problem 4.2. Suppose that $Y$ is p-periodic. Under what conditions does Eq.(2.1) have a r-periodic solution, where $r$ divides $p$ ? 


\section{$5 \quad$ Limiting equations}

Suppose that $f_{n}: D \rightarrow D$, where $D$ is a compact subset of $\mathbb{R}^{m}$, is a sequence of continuous functions that converges uniformly to a continuous function $f_{\infty}: D \rightarrow D$. Then the equation

$$
x_{n+1}=f_{\infty}\left(x_{n}\right)
$$

is called the limiting equation of the equation

$$
x_{n+1}=f_{n}\left(x_{n}\right)
$$

Open Problem 5.1. Suppose that $D=[a, b]$, where $[a, b] \subset \mathbb{R}$ is a compact interval, and that $f_{\infty}$ has a $2^{k}$-cycle but no $2^{k+1}$-cycles. Find conditions under which the omega-limit set of any point in Eq.(5.2) is a $2^{r}$-cycle of $f_{\infty}$, with $0 \leq r<k$.

Under a summability condition on the sequence $\left\{f_{n}\right\}$, Kempf [7] proved the conjecture for $k=1$. Hirsch, Smith, and Zhao [6] proved that the omegalimit of any point in Eq.(5.2) is internally chain transitive.

Open Problem 5.2. 1. Under what conditions does stability of cycles under $f_{\infty}$ can be lifted to the nonautonomous system?

2. Under what conditions does periodicity and almost periodicity can be lifted from $f_{\infty}$ to the nonautonomous system?

\section{References}

[1] S. Elaydi. Discrete Chaos, Chapman \& Hall, Boca Raton, 2000.

[2] S. Elaydi, On a converse of Sharkovsky's theorem, American Math. Monthly, 103 (1996) 386-392.

[3] S. Elaydi and R. Sacker, Global stability of periodic orbits of nonautonomous difference equations, Reprint.

[4] S. Elaydi and A. Yakubu, Global stability of cycles: Lotka-Volterra competition model with stocking, J. Diff. Equations and Appl. 8(6) (2002), 537-549. 
[5] S. Elaydi and A. Yakubu, Basins of attraction of stable cycles, J. Diff. Equations Appl. 8(8) (2002), 755-760.

[6] M.W. Hirsch, H.L. Smith, X-Q. Zhao, Chain transitivity, attractivity and strong repellors for semidynamical systems, J. Dynamics and Diff. Equations 13 (2001).

[7] R. Kempf, On $\Omega$-limit sets of discrete-time dynamical systems, J. Diff. Equations and Appl. 8(12) (2002), 1121-1131.

[8] S. Sacker and G. Sell, Lifting properties in skew-product flows with applications to differential equations, Memoirs Amer. Math. Soc. 11, No. 190 (1977).

[9] A. Sharkovsky, Coexistence of cycles of a continuous mapping of the line into itself (in Russian), Ukrain. Mat. Ž. 16 (1964), 61-71. 